\title{
Cytotoxicity induced by cypermethrin in Human Neuroblastoma Cell Line SH-SY5Y
}

\author{
Grzegorz Raszewski', Marta Kinga Lemieszek ${ }^{2}$, Krzysztof Łukawski ${ }^{1}$ \\ ${ }^{1}$ Department of Physiopathology, Institute of Rural Health, Lublin, Poland \\ ${ }^{2}$ Department of Medical Biology, Institute of Rural Health, Lublin, Poland
}

Raszewski G, Lemieszek MK, Łukawski K. Cytotoxicity induced by cypermethrin in Human Neuroblastoma Cell Line SH-SY5Y. Ann Agric Environ Med. 2016; 23(1): 106-110. doi: 10.5604/12321966.1196863

\begin{abstract}
The purpose of this study was to evaluate the cytotoxic potential of Cypermethrin (CM) on cultured human Neuroblastoma SH-SY5Y cells. SH-SY5Y cells were treated with CM at $0-200 \mu M$ for 24,48 , and $72 \mathrm{~h}$, in vitro. It was found that CM induced the cell death of Neuroblastoma cells in a dose- and time-dependent manner, as shown by LDH assays. Next, some aspects of the process of cell death triggered by CM in the human SH-SY5Y cell line were investigated. It was revealed that the pan-caspase inhibitor Q-VD-OPh, sensitizes SH-SY5Y cells to necroptosis caused by CM. Furthermore, signal transduction inhibitors PD98059, SL-327, SB202190, SP600125 failed to attenuate the effect of the pesticide. Finally, it was shown that inhibition of TNF-a by Pomalidomide (PLD) caused statistically significant reduction in CM-induced cytotoxicity. Overall, the data obtained suggest that CM induces neurotoxicity in SH-SY5Y cells by necroptosis.
\end{abstract}

\section{Key words}

Cypermethrin, cell death, necroptosis, mechanism

\section{INTRODUCTION}

Cypermethrin (CM) [(RS)-a-cyano-3-phenoxybenzyl(1RS)cis-trans-3-(2,2-dichloro-vinyl)-2,2-dimethylcyclopropa necarboxylate] is a synthetic type II pyrethroid which is extensively applied to control agricultural, as well as in consumer products for domestic purposes [1], due to their high insecticidal property and comparatively low toxicity.

Some studies have demonstrated that CM crosses the blood-brain barrier (BBB) and induces neurotoxicity [2, 3]. The neurotoxic responses of $\mathrm{CM}$ are mainly mediated by the modulation of ion channels. As one of the primary targets for CM is the voltage-gated sodium channel, CM extends the opening of sodium channels in the central nervous system leading to hypo-polarization and hyperexcitation of the neurons [4]. Moreover, it is known that short-term neurotoxicity caused by $\mathrm{CM}$ is primarily mediated through hyper-excitation of the central nervous system [3]. Additionally, CM induces neurotoxicity by modulating the glutamate receptors, acetylcholine receptors and ATP-ases [2]. Furthermore, CM-mediated neurotoxicity is contributed by its ability to induce the generation of reactive oxygen species (ROS) and DNA damage [5]. Oral or intra-peritoneal administration of CM produces oxidative stress in the neuronal system [6]. ROS, especially superoxide anion and hydrogen peroxide, are important signaling molecules not only in developing and proliferating cells, but also in the induction of apoptosis [7]. A recent study by Maurya et al. has shown that $\mathrm{CM}$ induces $\mathrm{Ca} 2+$ dependent activation of ROS, JNK1/2 and P38, causing disruption of BBB integrity in primary astrocytes [8]. A few reports have also shown the adverse effects of $\mathrm{CM}$ leading to nigrostriatal dopaminergic neurotoxicity $[3,9]$. The potential developmental neurotoxicity of CM has also been studied in dopaminergic SH-SY5Y

Address for correspondence: Grzegorz Raszewski, Department of Physiopathology Institute of Rural Health, Lublin, Jaczewskiego 2, 20-090 Lublin, Poland

E-mail: raszewskigj@gmail.com

Received: 02 November 2015; accepted: 12 January 2016 cells [10]. The decision taken by a cell to undergo apoptosis, autophagy and necroptosis is regulated by various factors, including the energy/ATP levels, the extent of damage or stress, and the presence of inhibitors of specific pathways. Our previous studies showed that the mixture of $\mathrm{CPF}$ and $\mathrm{CM}$ is more toxic to the SH-SY5Y cells than CPF used alone [11]. Hence, for the presented study, it was decided to examine the cytotoxicity of CM only, as well as some aspects of the process of cell death triggered by CM in the human SH-SY5Y cell line.

\section{MATERIALS AND METHOD}

Reagents. Cypermethrin [(RS)-a-cyano-3-phenoxybenzyl(1RS)-cis-trans-3-(2,2-dichloro-vinyl)-2,2-dimethylcycl opropanecarboxylate] was purchased (Fluka, Sigma-Aldrich, St. Louis, MO, USA). Pomalidomide (3-amino-thalidomide), MAPK inhibitors: PD98059, SL 327, SB202190, SP600125 and 3-(4,5-dimethylthiazolyl-2)-2,5-diphenyltetrazolium bromide (MTT), were also obtained from Sigma, a Pancaspase inhibitor Q-VD-OPh (N-(2-quinolyl)valyl-aspartyl(2,6-difluoro- phenoxy) methylketone) was purchased (BioVision, Mountain View, CA, USA), and a lactate dehydrogenase (LDH) kit also purchased (Roche, Basel, Switzerland). Unless otherwise stated, all other reagents were purchased from the Sigma-Aldrich Chemical Company.

Reagents working solutions. Cypermethrin was dissolved in ethanol to concentration $100 \mathrm{mM}$. Pan-caspase inhibitor, Q-VD-OPh $(1 \mathrm{mM})$ and all MAPK inhibitors $(10 \mathrm{mM})$ and Pomalidomide, PMD (10 mM) stock solutions were prepared in DMSO. Before performing the experiments, working solutions were prepared by dissolving an appropriate stock solution in culture medium. All solutions used in the experiments were prepared in medium supplemented with $2 \%$ FBS. Final ethanol and DMSO concentrations in medium did not exceed $0.05 \%$. 
Cell culture. Human undifferentiated neuroblastoma cell line SHSY5Y was purchased from ECACC (European Collection of Cell Cultures), Salisbury, UK. Cells were grown in 1:1 mixture of Ham's F12 nutrient and Dulbecco's modified Eagle's medium (DMEM) supplemented with $15 \%$ foetal bovine serum (FBS), 1\% non-essential amino acid solution, penicillin $(100 \mathrm{U} / \mathrm{mL})$ and streptomycin $(100 \mathrm{mg} / \mathrm{mL})$. Cells were maintained in a humidified atmosphere of $95 \%$ air and $5 \% \mathrm{CO} 2$ at $37^{\circ} \mathrm{C}$.

Cytotoxicity studies. As described previously [12], the cytotoxicity was estimated by measurement of Lactate Dehydrogenase (LDH) activity efflux from damaged cells into the medium. SH-SY5Y cells at $2 \times 10^{5}$ cells $/ \mathrm{mL}$ were treated with serial dilutions of CYP $(1-200 \mu \mathrm{M})$ for 24,48 and $72 \mathrm{~h}$, at $37^{\circ} \mathrm{C}$ in $5 \% \mathrm{CO} 2 . \mathrm{LDH}$ activity released from $\mathrm{SH}-\mathrm{SY} 5 \mathrm{Y}$ cells, which represents cell death (necrosis), was determined with an LDH kit (In vitro Toxicology Assay Kit Lactate Dehydrogenase Based, Sigma) according to the manufacturer's instructions.

Cytotoxicity was calculated by the following formula:

$$
\% \text { Cytotoxicity }=100 \times(E-S) \mid(M-S)
$$

where $\mathrm{E}$ is experimental LDH release from CM-treated cells; $\mathrm{M}$ is maximal $\mathrm{LDH}$ release caused by Triton $\mathrm{X}-100$, and $\mathrm{S}$ is spontaneous LDH release from untreated cells [13].

Inhibitory studies were conducted by incubating $\mathrm{SH}$ SY5Y cells $\left(2 \times 10^{5}\right.$ cells $\left./ \mathrm{mL}\right)$ with $50 \mu \mathrm{lM}$ CM in the presence and absence of $5 \mu \mathrm{M}$ Q-VD-OPh, $20 \mu \mathrm{M}$ PD98059 (ERK inhibitor), SL-327 (MEK inhibitor), SB202190 (p38 MAPK inhibitor), SP600125 (JNK inhibitor). After 48h of incubation in standard conditions, cytotoxicity of CM to SH-SY5Y cell after treatment using the $\mathrm{LDH}$ release were determined, as described above.

Statistical analysis. The data were presented as the mean value and standard error of the mean (S.E.M.). Statistical analysis was performed with the one-way ANOVA with Tukey's post hoc test (GraphPad Prism 5 software package, version 5.02, GraphPad Software Inc. USA). Significance was accepted at $\mathrm{p}<0.05$.

\section{RESULTS}

1. Cypermethrin induces cytotoxicity in SH-SY5Y cell cultures. To examine the toxic effects of CM, SH-SY5Y cells were treated with varying concentrations of CM and assayed for cell viability at various times after treatment using the $\mathrm{LDH}$ leakage from the SH-SY5Y cells into the incubation medium. Measuring LDH release is a useful method for detection of necrosis [12]. Treatment of cells with 1, 10, 25, 50, 100, $200 \mu \mathrm{M} \mathrm{CM}$, at 24, 48 and $72 \mathrm{~h}$, caused significant increases of cytotoxicity against SH-SY5Y cells (Tab. 1). The cytotoxic effects were dose- and time-dependent manner.

Our subsequent experiments with CM were carried out at $50 \mu \mathrm{M}$, which caused $45.9 \pm 3.26 \%(\mathrm{p}<0.001)$ cytotoxicity to SH-SY5Y, after $48 \mathrm{~h}$ treatment.

2. Mechanisms of CM toxicity in SH-SY5Y cell cultures. To investigate the mechanism by which CM induces cytotoxicity in SH-SY5Y cells, we examined the influence of inhibitors of proteins involved in cell death.

To determine whether the major caspase pathways are involved in the regulation of CM-induced cytotoxicity, SHSY5Y cells were exposed to $50 \mu \mathrm{M}$ CM in the presence and absence of $5 \mu \mathrm{M}$ Q-VD-OPh, a pan-caspase inhibitor, for 48h. Q-VD-OPh slightly enhanced $(\mathrm{p}=0.0329) \mathrm{CM}$-induced cytotoxicity in SH-SY5Y cell cultures (Fig. 2). In addition, the dose of $5 \mu \mathrm{M}$ Q-VD-OPh was not toxic against tested cells. Thus, these data suggest that Q-VD-OPh sensitizes SH-SY5Y cells to necrosis caused by CM.

It is known that tumour necrosis factor (TNF) mediates apoptotic as well as necrotic forms of cell lysis; therefore, this study aimed to determine whether its activation mediates $\mathrm{CM}$-induced cytotoxicity. For this purpose, CM-treated cells were exposed to Pomalidomide (PLD), pharmacological inhibitor of TNF-a production. The treatment with $25 \mu \mathrm{g} / \mathrm{mL}$ PLD cause slightly but statistically significant $(\mathrm{p}=0.00345)$ reduction in CM-induced cytotoxicity (Fig. 3).

To ascertain which MAP kinase signaling pathways mediated the toxic effects of CM, SH-SY5Y cells were exposed to $50 \mu \mathrm{M}$ pesticide in the presence $(20 \mu \mathrm{M})$ and absence of several specific inhibitors of these signaling pathways: PD98059 (ERK inhibitor), SL-327 (MEK inhibitor), SB202190 (p38 MAPK inhibitor) and SP600125 (JNK inhibitor), for 24h.
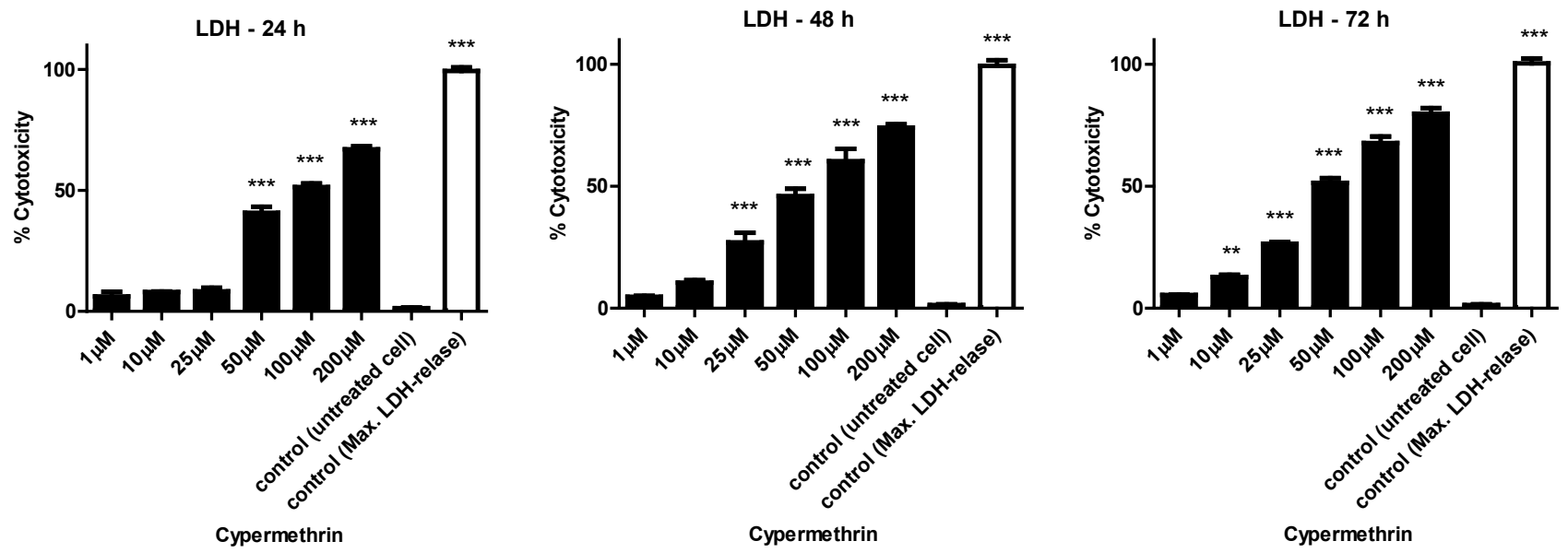

Figure 1. Cypermethrin (CM) causes dose-dependent SH-SY5Y cell cytotoxicity. Neuroblastoma cells were incubated for 24,48 and $72 \mathrm{~h}$ in serum-free media containing CM $(0-200 \mu \mathrm{M})$, and Lactate dehydrogenase (LDH) ELISA kit was used to quantify LDH release and percentage of cytotoxicity was measured, as described above. Data are representative of three independent experiments. Statistical analysis was performed with one-way ANOVA. ${ }^{* * *} p<0.001 ;{ }^{* *} p<0.01$ when compared with controls 


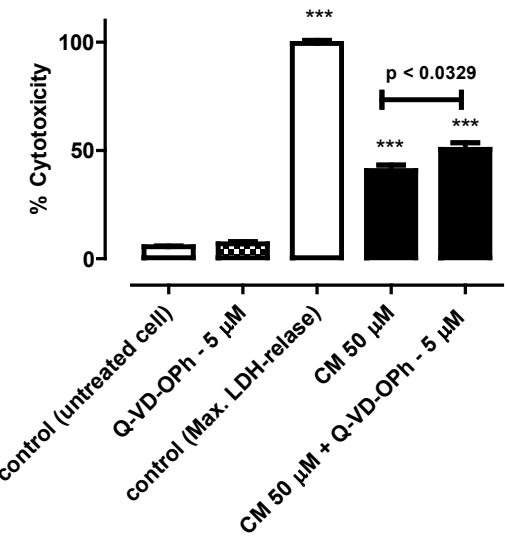

Figure 2. Effect of Q-VD-OPh (pan-caspase inhibitor) on cypermethrin (CM)induced SH-SY5Y cell cytotoxicity. SH-SY5Y cells were pre-incubated with $5 \mu \mathrm{M}$ Q-VD-OPh for $1 \mathrm{~h}$ before treatment with CM for $48 \mathrm{~h}$. A Lactate dehydrogenase (LDH) ELISA kit was used to quantify LDH release and percentage of cytotoxicity was measured as described above. Data are representative of three independent experiments. Statistical analysis was performed with one-way ANOVA. ${ }^{* * *} p<0.001$ when compared with controls.

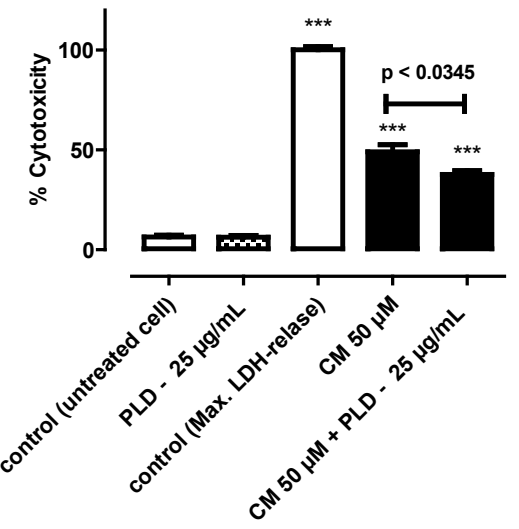

Figure 3. Effect of Pomalidomide (PLD), (pharmacological inhibitor of TNF-a production) on cypermethrin (CM)-induced SH-SY5Y cell cytotoxicity. SH-SY5Y cells were pre-incubated with $25 \mu \mathrm{g} / \mathrm{mL}$ PLD for $1 \mathrm{~h}$ before treatment with $\mathrm{CM}$ for 48h. A Lactate dehydrogenase (LDH) ELISA kit was used to quantify LDH release and percentage of cytotoxicity was measured as described above. Data are representative of three independent experiments. Statistical analysis was performed with one-way ANOVA. ***p $<0.001$ when compared with controls
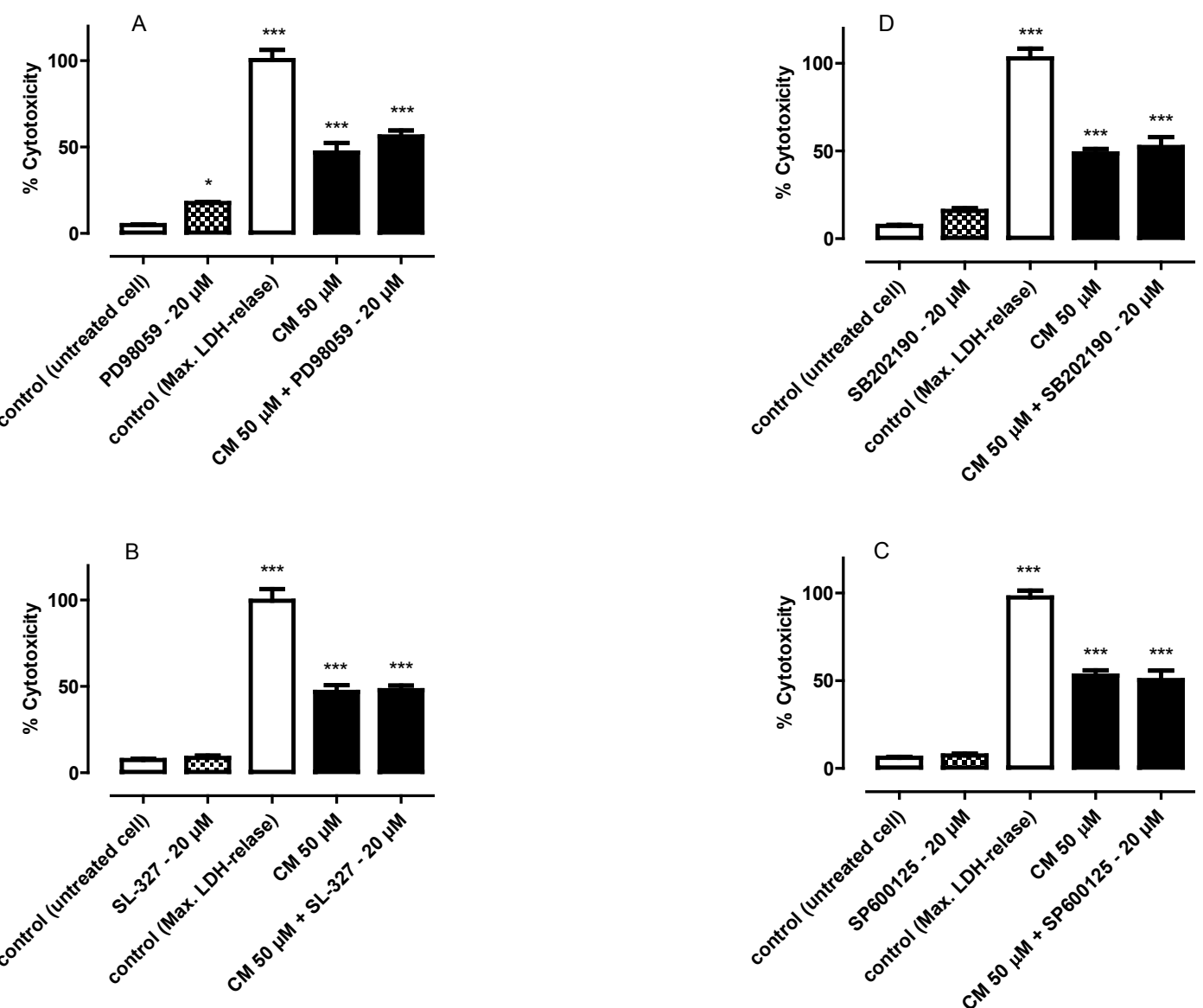

Figure 4. Effects of signal transduction inhibitors on cypermethrin (CM)-induced SH-SY5Y cell cytotoxicity. SH-SY5Y cells were pre-incubated in the presence and

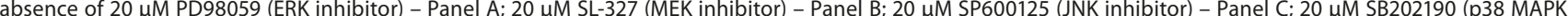
inhibitor) - Panel D, for $1 \mathrm{~h}$ before treatment with $\mathrm{CM}$ for $48 \mathrm{~h}$. A Lactate dehydrogenase (LDH) ELISA kit was used to quantify LDH release and percentage of cytotoxicity as described above. Data are representative of three independent experiments. Statistical analysis was performed with one-way ANOVA. Data are representative of three independent experiments. ${ }^{* *} \mathrm{p}<0.001$ when compared with controls.

As shown in fig. 3, PD98059, SL-327, SP600125 and SB202190 failed to alter the toxic effects of CM. However, SH-SY5Y cells exposed to PD98059 exhibited enhanced cellular toxicity (Fig. 4).

\section{DISCUSSION}

Necrosis is a type of cell death that is morphologically characterized by swelling and rupture of intracellular organelles, eventually leading to the breakdown of the 
plasma membrane. The cytotoxicity assays for measuring necrosis are based on the leakage of intracellular molecules through impaired plasma membrane [13]. LDH is a soluble cytoplasmic enzyme that is present in almost all cells and is released into extracellular space when the plasma membrane is damaged by stress, injury, chemicals or intercellular signals. Thus, the quantification $\mathrm{LDH}$ from cells is one of the major methods to assess cell death by necrosis [14]. Necrosis has been defined as a type of cell death that lacks the features of apoptosis and is usually considered to be uncontrolled. However, several recent studies have clearly established that its occurrence and course might be tightly regulated [15].

The presented study examines whether exposure to CM results in cytotoxicity by assessing $\mathrm{LDH}$ leakage from the SH-SY5Y to the incubation medium. Treatment of cells with $0-200 \mu \mathrm{M}$ of CM produced significant elevations in $\mathrm{LDH}$ leakage, and induced cytotoxicity in concentration- and time-dependent ways (Fig. 1).

The cytotoxic effect of Ripcort (a preparation contained an active compound cypermethrin) on SH-SY5Y cells was examined previously by Kokko et al. [16] in which exposure to $0.1-100 \mu \mathrm{M}$ of $\mathrm{CM}$ showed dose-dependent cytotoxicity. The detectable toxicity of CM started at $1 \mu \mathrm{M}$ concentration and at a concentration of $15 \mu \mathrm{M}$ the cell viability (WST1) was about $72 \%$ of control, while at $25 \mu \mathrm{M}$ decreased to $15 \%$ of control. Unfortunately, in this work, CM was used with a mixture of xylene and petrol $(820 \mathrm{~g} / \mathrm{L})$ and the toxic effects of these all substances could be additive. In earlier work [11], CM used in concentrations from 0.5-5 $\mu \mathrm{M}$, for $24 \mathrm{~h}$, had no effect on SH-SY5Y cell viability as determined by MTT assay, and these observations are consistent with the data on cytotoxicity obtained by LDH leakage assay in the presented study. Additionally, CM had a significantly synergistic effect on CPF-induced cytotoxicity in studied cells [11]. Similarly, some studies showed that CM reduced the viability of astrocytes [8] and rat hepatocytes [17] in a dose-dependent manner.

It is known that necrosis can also function as an alternative programmed mode of cell death, triggered by the same death signals that induce apoptosis. The term 'necroptosis' was later introduced to describe the cases where necrosis represented a regulated and programmed form of death [18].

Apoptosis may be triggered either by extrinsic stimuli through cell surface death receptors, such as TNFa (tumour necrosis factor- $\alpha$ ), Fas (CD95/APO1) and TRAIL (TNF related apoptosis inducing ligand), or by intrinsic stimuli via the mitochondrial signaling pathway [18]. The death receptors that typically induce apoptosis, have also been clearly shown to induce necroptosis in different cell types [18]. It is known that TNF-a signaling is mediated via two distinct receptors, TNFR1 and TNFR2, which showed partially overlapping signaling mechanisms and biological roles depending on cell type. Contrary to the cytotoxic effects of TNF-a through TNFR1, there is substantial evidence showing that TNF-a can promote neural cell survival through another TNF-a receptor, TNFR2 via the activation of $\mathrm{NF}_{\mathrm{k}} \mathrm{B}$ [19].

The signal transduction cascade of TNFa leading to apoptotic and necroptotic cell death has been studied in detail $[20,21]$. The binding of TNFa to TNFR1 leads to its internalization and the formation of a cytosolic death-inducing signaling complex (DISC), also known as complex II. By contrast, when caspase- 8 is deleted, depleted or inhibited, complex II cannot initiate the apoptotic programme and ligation of TNFR1 results in necroptosis [22]. As shown, in response to TNF- $\alpha$ antigen stimulation, autophagy is activated to blocking necroptosis in several cell lines, such as L929 cells, lymphocytes and cancer cells [18]. These results were confirmation that a caspases inhibitor can induce necroptosis and prevent autophagy, underscores the pro-survival function of autophagy against necroptosis. Both autophagy and apoptosis are well-controlled biological processes that play essential roles in development, tissue homeostasis and disease. Moreover, autophagy is induced as an adaptive response against endoplasmic reticulum (ER) stress [23]. A recent study, carried out in the SH-SY5Y cell line, demonstrated that Chlorpyrifos (CPF)-induced cytotoxicity is modified by autophagy regulation, and that rapamycin protects against CPF-induced apoptosis by enhancing autophagy [24]. It was found that treatment with $\mathrm{Q}-\mathrm{VD}-\mathrm{OPh}$, pan-caspase inhibitor, caused a slight increase $(\mathrm{p}=0.0329)$ in CM-induced cytotoxicity in SH-SY5Y cell cultures. These observations were confirmation of the results obtained in the current study.

Pomalidomide (PMD) is a third-generation immunomodulatory drug with significant activity in multiple myeloma. Its mechanism of action is incompletely understood, but concerns anti-angiogenic and other unknown antiinflammatory effects [25]. In addition, PMD is the most potent in inhibition of TNF- $\alpha$ production [25]. An earlier study by the authors of the presented study showed that PMD attenuated CPF-induced apoptosis in SHSY5Y cells. Also, in this study, CM given in a dose of 2.5uM had no effect on the cytotoxicity of CPF to tested cells [11].

In the presented study, preincubation with PMD resulted in a decrease of cytotoxicity of $50 \mu \mathrm{M}$ CM against SH-SY5Y cells. These results show that PMD, the inhibitor of TNF- $\alpha$ production may cause down-regulation of the TNFR1 receptors, and decrease of CM induced-cytotoxicity in $\mathrm{SH}$ SY5Ycells.

This study also shows that none of the signal transduction inhibitors reversed the toxic effects of CM on studied cells at $24 \mathrm{~h}$ exposure. On the other hand, the expression of MAPKs in SH-SY5Y cells was not investigated, although the activation of MAPK-s after exposure to CM has been demonstrated in some studies. In a study by Agrawal et al., CM (at a dose of $15 \mathrm{mg} / \mathrm{kg}$, twice a week for 12 weeks) induced the activation of JNK, caspase-3, tumour suppressor protein (p53), TNF-a, p38 MAPK in the nigrostriatal dopaminergic neurons in rats [28]. Similarly, CM induced astrocyte injury via modulation in ROS, JNK and P38 pathways [8]. The results presented are not consistent with these data, but the experiments for the presented study were carried out in a different experimental model. On the other hand, the expression of MAPKs in SHSY5Y cells were also not examined.

\section{CONCLUSION}

The presented study provides insights concerning the molecular mechanisms of CM-induced cytotoxicity in human Neuroblastoma SH-SY5Y cells. CM was found to induce neurotoxicity in SH-SY5Y cells via necroptosis signaling pathways. The obtained results may have implications for estimating the risks of cypermethrin exposure. 


\section{Acknowledgement}

This work was supported by the National Science Centre, Krakow, Poland, Grant Number N N404 519338.

\section{REFERENCES}

1. Costa LG, Giordano G, Guizzetti M, Vitalone A. Neurotoxicity of pesticides: a brief review. Front Biosci. 2008; 13: 1240-1249.

2. Gupta A, Agarwal AK, Shukla GS. Effect of quinalphos and Cypermethrin exposure on developing blood-brain barrier: role of nitric oxide. EnvironToxicol Pharmacol. 2000; 2: 73-78.

3. Singh AK, Tiwari MN, Prakash O, Singh MP. A current review of cypermethrin-induced neurotoxicity and nigrostriatal dopaminergic neurodegeneration. Curr Neuropharmacol. 2012;10(1): 64-71.

4. Soderlund D. Molecular mechanisms of pyrethroid insecticide neurotoxicity: Recent advances. Arch Toxicol. 2012; 86: 165-181.

5. Hsieh HL, Wang HH, Wu WB, Chu PJ, Yang CM. Transforming growth factor-betal induces matrix metalloproteinase- 9 and cell migration in astrocytes: roles of ROS-dependent ERK- and JNK-NF-kappaB pathways. J Neuroinflammation. 2010; 7: 1-17.

6. Tiwari MN. Singh AK, Israr A, Upadhyay G, Singh D, Patel DK, et al. Effects of cypermethrin on monoamine transporters, xenobiotic metabolizing enzymes and lipid peroxidation in the rat nigrostriatal system. Free Radic Res. 2010; 44: 1416-1424.

7. Gutteridge JM, Halliwell B. Antioxidants: molecules, medicines, and myths. Biochem Biophys Res Commun. 2010; 393: 561-564.

8. Maurya SK, Rai A, Rai NK, S. Deshpande S, Jain R, Mudiam MK, et al. Cypermethrin induces astrocyte apoptosis by the disruption of the autocrine/paracrine mode of epidermal growth factor receptor signaling. Toxicol Sci. 2012; 125: 473-487.

9. Mun JY, Lee WY, Han SS. Effects of cypermethrin on the dopaminergic neurons in the progressive hemiparkinsonian rats. Toxicol Mech Methods. 2005; 15: 399-404.

10. Flaskos J, Harris W, Sachana M, Munoz D, Tack J, Hargreaves AJ. The effects of diazinon and cypermethrin on the differentiation of neuronal and glial cell lines. Toxicol Appl Pharmacol. 2007; 219: 172-80.

11. Raszewski G, Lemieszek MK, Łukawski K, Juszczak M, Rzeski W. Chlorpyrifos and cypermethrin induce apoptosis in human neuroblastoma cell line SH-SY5Y. Basic Clin Pharmacol Toxicol. 2015; 116(2): 158-67.

12. Koh JY, Choi DW. Quantitative determination of glutamate mediated cortical neuronal injury in cell culture by lactate dehydrogenase efflux assay. J Neurosci Methods. 1987; 20: 83-90.
13. Chan FK, Moriwaki K, De Rosa MJ. Detection of necrosis by release of lactate dehydrogenase activity. Methods Mol Biol. 2013; 979: 65-70.

14. Moquin D, Chan FK. The molecular regulation of programmed necrotic cell injury. Trends Biochem Sci. 2010; 35: 434-441.

15. Golstein P, Kroemer G. Cell death by necrosis: towards a molecular definition. Trends Biochem Sci. 2007; 32(1): 37-43.

16. Kakko I, Toimela T, Tähti H. The toxicity of pyrethroid compounds in neural cell cultures studied with total ATP, mitochondrial enzyme activity and microscopic photographing. Environ Toxicol Pharmacol. 2004; 15: 95-102.

17. Grajeda-Cota P, Ramírez-Mares MV, González de Mejía E. Vitamin C protects against in vitro cytotoxicity of cypermethrin in rat hepatocytes. Toxicol In Vitro. 2004; 18(1): 13-9.

18. Nikoletopoulou V, Markaki M, Palikaras K, Tavernarakis N. Crosstalk between apoptosis, necrosis and autophagy. Biochim Biophys Acta. 2013; 1833(12): 3448-59.

19. Mitsiades N, Mitsiades C, Poulaki V, Chauhan D, Richardson P, Hideshima T, et al. Apoptotic signaling induced by immunomodulatory thalidomide analogs in human multiple myeloma cells: therapeutic implications. Blood 2002; 99: 4525-4530

20. He S, Wang L, Miao L, Wang T, Du F, Zhao L, et al. Receptor interacting protein kinase-3 determines cellular necrotic response to TNF-alpha. Cell 2009; 137: 1100-1111.

21. Zhang DW, Shao J, Lin J, Zhang N, Lu BJ, Lin SC, et al. RIP3, an energy metabolism regulator that switches TNF-induced cell death from apoptosis to necrosis. Science 2009; 325: 332-336.

22. Holler N, Zaru R, Micheau O, Thome M, Attinger A, Valitutti S, et al. Fas triggers an alternative, caspase-8-independent cell death pathway using the kinase RIP as effector molecule. Nat Immunol. 2000; 1: 489-495.

23. He C, Klionsky DJ. Regulation mechanisms and signaling pathways of autophagy, Annu Rev Genet. 2009; 43: 67-93.

24. Park JH, Lee JE, Shin IC, Koh HC. Autophagy regulates chlorpyrifosinduced apoptosis in SH-SY5Y cells. Toxicol Appl Pharmacol. 2013; 268: 55-67.

25. Zhu YX, Kortuem KM, Stewart AK. Molecular mechanism of action of immune-modulatory drugs thalidomide, lenalidomide and pomalidomide in multiple myeloma. Leuk Lymphoma. 2013; 54(4): 683-7.

26. Skulachev VP. Bioenergetic aspects of apoptosis, necrosis and mitoptosis, Apoptosis 2006; 11: 473-485.

27. Amaravadi RK, Thompson CB. The roles of therapy-induced autophagy and necrosis in cancer treatment. Clin Canc Res. 2007; 13: 7271-9.

28. Agrawal S, Singh A, Tripathi P, Mishra M, Singh PK, Singh MP. Cypermethrin-induced nigrostriatal dopaminergic neurodegeneration alters the mitochondrial function: a proteomics study. Mol Neurobiol. 2015; 51(2): 448-465. 